\title{
Polygenic Risk Scores and Bipolar Disorder
}

\author{
Susanne Bengesser *, Eva Reininghaus \\ Department of Psychiatry and Psychotherapeutic Medicine, Medical university \\ of Graz (MUG), Auenbruggerplatz 31, 8036 Graz, Austria.
}

* Corresponding Author: Susanne Bengesser. Email:

Susanne.Bengesser@medunigraz.at.

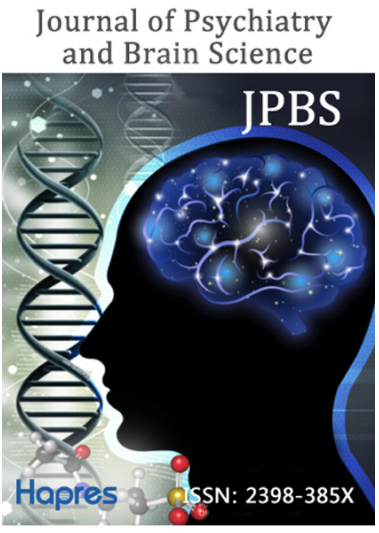

https://jpbs.hapres.com

\section{f OPEN ACCESS}

DOI: $10.20900 / j p b s .20180013$

Received: October 29, 2018

Accepted: December 18, 2018

Published: December 21, 2018

Copyright: $\odot 2018$ by the authors. Licensee Hapres, London, United Kingdom. This is an open access article distributed under the terms and conditions of the Creative Commons Attribution License (CC BY 4.0, https://creativecommons.org/licenses/by/4.0/).

\begin{abstract}
Bipolar disorder is an affective disorder with mood swings between the pole of depression and the pole of mania. The mechanism of those mood swings has not been completely elucidated yet. According to current scientific knowledge affective episodes are caused by a concatenation between a genetic predisposition and biopsychosocial triggers. Even though diverse genome wide associated gene variants (e.g., single nucleotide polymorphisms of CACNA1C, ANK3 or NCAN) were associated with Bipolar disorder in the last decade, there is still missing heritability. Thus, innovative approaches and endophenotypes of Bipolar disorder must be used to decipher the underlying disease mechanisms of Bipolar disorder. Recently, the Polygenic Risk Score for Schizophrenia was investigated in the lithium response endophenotype of Bipolar disorder. The polygenic score for Schizophrenia was inversely associated with the lithium response phenotype in study participants with Bipolar disorder. Furthermore, there are genetic overlaps between psychiatric diseases, which also demonstrates the importance of cross-disorder design studies to elucidate the missing heritability of Bipolar disorder.
\end{abstract}

Keywords: bipolar disorder; polygenic risk score; schizophrenia

\section{INTRODUCTION}

Bipolar Disorder (BD) is an affective disorder, which is characterized by mood swings between the pole of mania and depression. The BD spectrum disorders include BD type I (recurrent depressive and manic episodes), BD type II (recurrent depressive and hypomanic episodes, 
but no episodes of mania) and cyclothymia (over at least two years dysthymic mood with mild hypomanic episodes in between) ${ }^{[1]}$. Mood disorders are widespread diseases, which go in line with decreased quality of life, increased cardiovascular risk and increased mortality ${ }^{[2,3]}$. The lifetime prevalence lies around 3\% according to the German S3 guidelines for BD, but there is still a significant number of unreported cases ${ }^{[4]}$. BD leads to increased individual suffering and unfortunately a major proportion of patients (around 30\%) are not responding completely to the currently available state of the art therapy (e.g., the mood stabilizer lithium) ${ }^{[4,5]}$. Deciphering the molecular blueprint of BD is therefore important to find new drug targets and to improve already existing treatment strategies ${ }^{[6,7]}$.

Twin and adoption studies showed very early the high hereditary factor of BD. The concordance rates between monozygotic twins lie around $80 \%$, which underlines the strong genetic predisposition of $B D^{[1]}$. In the last decades geneticists have discovered hundreds of potential candidate genes with the early linkage- and hypothesis driven gene association studies and more recently with modern genome wide association studies (GWAS), which are summarized by diverse comprehensive already published reviews ${ }^{[6,7]}$. Since this current narrative review is focussing on the polygenic risk score in $\mathrm{BD}$, only some historically important candidate gene stories are reviewed at the beginning:

The first candidate gene approaches in the field of BD focused on genes involved in pathways that fit in the scientific models of affective disorders ${ }^{[8-35]}$. Diverse hypothesis driven gene association studies investigated the serotonergic system (e.g., SERT), the dopaminergic system (e.g., DRD 1-5, DAT1 syn. SLC6A3), mechanisms of neurotransmitter breakdown (e.g., MAOA, COMT), glutamatergic neurotransmitter system (e.g., GRM7), GABAergic neurotransmitter system (e.g., GABRBI, GABRB3, GABRB5), growth factors (e.g., BDNF) and circadian rhythms (e.g., ARNTL syn. BMAL1) ${ }^{[6]}$. More in detail, $B D N F$ (Brain derived neurotrophic factor), encoded on 11p13-15, has several important functions like neuronal survival, migration, myelination, phenotypic differentiation, neurogenesis, axonal and dendritic growth, synapse formation, learning and memory formation, as well as antidepressant effects ${ }^{[8-17]}$. Le-Niculescu and colleagues ${ }^{[18]}$ discovered BDNF as one of the top candidate genes for manic-depressive disorder in a functional re-analysis of GWAS ${ }^{[8,12,18]}$. The Serotonin-transporter gene (SERT) was another highly investigated historic candidate gene. The deletion/insertion polymorphism in the promoter region of SERT was associated with BD in an early metaanalysis by Lasky and colleagues including 2,774 subjects with BD and 3,652 controls ${ }^{[19]}$. The short allele (s-allele), which contains the deletion, leads to reduced transcriptional activity of the promoter, which consequently reduces the rate of expression of the serotonin transporter by $30-40 \%{ }^{[20,21]}$. COMT, another historically important candidate gene on 22q11.1-q11.2 codes for the Catechol-O-methyltransferase, which inactivates catecholamine transmitters ${ }^{[36]}$. The deletion on 22q11 leads to the velocardiofacial syndrome, an autosomal dominant congenital disorder associated with cleft palate, cardiac defects and learning disabilities. Because of very common comorbidity (up to $80 \%$ ) with schizophrenia, BD or ADHD (attention deficit hyperactivity disorder) the region reached increased interest in early eras of Psychiatric Genetics research ${ }^{[22]}$. Similar, MAOA encodes for the monoamine oxidase $A$ that catalyses the oxidative deamination of monoamines. Monoamine oxidase A breaks down serotonin, melatonin, noradrenaline and adrenaline, which explains the intensive research on MAOA gene variants in early hypothesis driven genetic analyses ${ }^{[23-29]}$. The clock genes are further interesting functional relevant BD candidate genes, which have been investigated for decades in hypothesis driven gene-association studies. Disturbed circadian rhythms (e.g., jetlag, shift work, sleeping disorders) lead to disturbed mood, while sleep deprivation therapy leads to fast brightening of the mood. Mood-, activity- and sleep diaries (either with paper and pen or with modern apps) can support long term mood stabilization. On a molecular biological basis, a complex transcriptionaltranslational feedback loop generates an almost $24 \mathrm{~h}$ rhythm and manages diverse important body functions in a circadian manner (e.g., hormone release, heartbeat, blood pressure). Even the mood itself is linked with the molecular clock according to a model postulated by Hampp and colleagues ${ }^{[37]}$. The gene product of the clock gene ARNTL activates as heterodimer with NPAS2 the transcription of the MAOA gene, which encodes for the serotonin, noradrenalin and dopamine degrading enzyme monoamine oxidase $A^{[37]}$. Many association studies already prove a connection between individual SNPs (Single Nucleotide Polymorphisms) of ARNTL and BD, while others could not replicate this finding recently ${ }^{[30-35]}$. ARNTL showed also significantly higher methylation at cg05733463 in individuals with BD compared to controls in a sample from Graz, Austria ${ }^{[38]}$. The association between circadian rhythms and mood was also found in a recent large scale GWAS of circadian rhythmicity in 71,500 UK Biobank participants and polygenic association of circadian rest-activity rhythmicity with mood instability, MDD (Major Depressive Disorder) and neuroticism ${ }^{[39]}$.

Despite those promising findings, candidate gene approaches failed to predict the risk for BD itself, even though the complete collection of all hypothesis 
driven genetic studies helps to paint a picture of the disease (for a review see ${ }^{[6]}$ ). Nevertheless, the decade of the brain led to improvement of genetic methods and nowadays there are more specialized means to analyse polygenic risk for mood disorders. Most important is the fact, that the genetic predisposition for $\mathrm{BD}$ is polygenic and each risk gene has a very low risk itself. Only the orchestra of susceptibility genes can lead to a certain vulnerability for the disease. It is current scientific consent that the disease is based on the vulnerability-stress-model and that the underlying genetic predisposition is particularly polygenic. Multinational, collaborative GWAS consortia were necessary to further elucidate the genetic underpinning of mood disorders. The first large PGC GWAS of the $\mathrm{BD}$ working group included 7,481 individuals with $\mathrm{BD}$ and 9,250 healthy controls, as well as a replication study of 4,496 independent BD individuals and 42,422 independent healthy controls. The primary PGC GWAS showed genome wide significant association of ANK3 (located on 10q21) for the imputed SNP rs10994397 $\left(p=7.1 \times 10^{-9}\right)$ and the SYNE1 rs9371601 $\operatorname{SNP}\left(p=4.3 \times 10^{-8}\right)$, which encodes for a synaptic nuclear envelope protein 1 . The new gene ODZ4 gene variant was also associated with $\mathrm{BD}$ in the primary GWAS $\left(p=2.1 \times 10^{-7}\right)$. ODZ4 (encoded on 11q14) is a human homologue of the Drosophila pair-rule gene odz. The combined analysis by Sklar et al. (63,766 study participants) showed genome-wide significant association of rs4765913 in CACNA1C $\left(p=1.82 \times 10^{-9}, \mathrm{OR}=1.14\right)$ and $\mathrm{rs} 12576775$ in ODZ4 $\left(p=2.77 \times 10^{-8} ; \text { OR }=0.89\right)^{[40]}$. Diverse large collaborative, international GWAS showed replicative genome wide significant association of CACNA1C, ANK3, ODZ4, DGKH, NCAN with BD, which underlines the importance of those genes in the disease mechanisms of $\mathrm{BD}{ }^{[6,7,40-48]}$. Ground breaking results were now obtained from the latest and largest BD PGC GWAS, which included 20,352 individuals with BD and 31,358 controls, as well as a follow up sample of the top findings in an independent sample of 9,412 bipolar individuals and 137,760 controls. Over 30 genome wide significant associated SNPs were associated with $\mathrm{BD}$, which underlined again the importance of ion channel subunits or ion channel associated proteins, neurotransmitter transporters, synaptic components, immune-inflammatory pathways and energy metabolism in the disease mechanisms of BD. CACNA1C, GRIN2A, SCN2A, SLC4A1, RIMS1 and $A N K 3$ belonged to those genome wide significant hits ${ }^{[41]}$. The historically mentioned candidate gene variants BDNF, SERT, COMT and MAOA did not belong to the genome wide significant results of this recent PGC GWAS, which highlights that hypothesis driven approaches may lead to missing the true associated risk genes. Nevertheless, still too small sample sizes can also lead to false negative results ${ }^{[41]}$.

\section{POLYGENIC RISK SCORES CAN PREDICT THE RISK FOR BD BETTER THAN SINGLE CANDIDATE GENES ALONE!}

The genetic architecture of psychiatric diseases is particularly polygenic and the polygenic risk score is based on a variation of genetic loci, which are jointly the best for the prediction of the trait. The polygenic scores are having even more predictive value than the genome wide statistically hits in genome wide association studies (GWAS) alone, because each SNP (Single Nucleotide Polymorphism) has only a rather small impact by itself. Since BD is based on a "kaleidoscope" of genetic variants, which may even include hundreds of gene variants ${ }^{[6]}$ with rather small effects by themselves, polygenic scores seem to be a precious measure of genetic prediction of the trait ${ }^{[49,50]}$. As GWAS collaborations are growing faster and faster, the accuracy of risk prediction is getting better and better, because the sample size of the discovery data is the most important factor for prediction of a trait value in an independent sample ${ }^{[49-51]}$. Nevertheless, even though SNPs (Single Nucleotide Polymorphisms) associated with Bipolar Disorder (BD) are considered jointly to calculate polygenic risk scores for this mood disorder, the individual risk prediction is still rather low and there is still hidden genetic heritability ${ }^{[49-51]}$. According to Dudbridge et al. hundreds of thousands of subjects would be needed to estimate the gene effects for the use of predicting individual risk of disease ${ }^{[49]}$. Therefore, the PGRS will not be used for individual genetic testing at Human Genetics Departments in the near future. Further research in strong-minded collaborations are still necessary to further increase joined GWAS sample sizes and replication samples to increase the power to identify still hidden risk genes with low effects and to further improve the PGRS (Polygenic Risk Score) in training samples. Until then the PGRS is still an important method to include a set of genes, which are likely to be involved in BD disease mechanisms according to well established models of affective disorders, but have not yet reached genome wide significance based on limited power. Polygenic scores are used to summarize genetic effects of an orchestra of genetic markers, which did not individually achieve genome wide significance in a large-scale association study. Those genetic markers are, as mentioned before, nominated by using training samples and by applying the constructed score in an independent replication sample by making the weighted sum of associated alleles within each subject. Hence, the PGRS can be used to find evidence of a genetic effect even when no single markers are significant on their own. This 
approach can be useful to establish also a shared genetic basis for related traits and to construct risk prediction models for psychiatric diseases in the field of research ${ }^{[49]}$. Increasing sample sizes of collaborative GWAS consortia will help to increase the accuracy of trait prediction with polygenic scores and will therefore get a step ahead towards precision medicine ${ }^{[49,51]}$.

\section{THERE IS STILL MISSING HERITABILITY IN BIPOLAR DISORDER (BD) GENETICS!}

As depicted in the last paragraphs, only the concatenation between the genetic vulnerability and diverse gene-environment-interactions are causing (BD), but not all disease mechanisms are known yet and there is still missing heritability ${ }^{[6]}$. Eli Stahl et al. reported in their latest report that only around $30 \%$ of heritability can be explained by SNP (Single Nucleotide Polymorphism)-heritability, which is not yet reached by previous research results (currently only around $8 \%$ are explained) and that therefore new approaches and further increasing sample sizes are necessary to further elucidate the genetic architecture of $\mathrm{BD}^{[41]}$.

To decipher the genetic predisposition of the mood disorder, diverse endophenotypes of BD have been investigated over the years with different genetic approaches. In this narrative review, we will present and discuss the genetic underpinning of BD with an emphasis on the PGRS of schizophrenia and BD. The literature search was performed in PubMed to identify genetic studies in the field of BD. Since hundreds of candidate-gene and genome wide association studies exist ${ }^{[6]}$, this review can only report excerpts of the genetic studies based on a subjective evaluation of their relevance for the field.

Narrowing down the phenotype is one approach to better investigate polygenic risk for BD. Since common genome wide association studies (GWAS) and candidate gene approaches have not completely deciphered the genetic architecture of BD, narrowing the phenotypes may improve the identification of risk genes that confer to special BD subtypes e.g., rapid cycling, BD type I versus BD type II, chronotypes, lithium response or psychotic features ${ }^{[49]}$.

The more we know about genetics and molecular biology of affective disorders, the more we know that the classic postulated boundaries of Psychiatry do not exist completely anymore. Especially the dichotomy postulated by Kraepelin and followers, which strictly divided affective disorders and schizophrenia, is nowadays blurred. Kraepelin divided strictly "manic depression" and "dementia praecox" (schizophrenia) ${ }^{[7]}$. Nowadays, the boundaries are fading and there is a major part of well replicated risk genes, which are shared between MDD, BD and schizophrenia e.g., the well replicated risk genes NCAN, ANK3, CACNA1C etc. ${ }^{[6,7]}$.

For this reason, in the next lines, we want to focus on selected shared risk genes. NCAN (neurocan) is a shared risk gene between $\mathrm{BD}$ and schizophrenia ${ }^{[52]}$ and encodes for an extracellular glycoprotein that is involved in adhesion, migration and cortical folding ${ }^{[52]}$. NCAN belongs to the best replicated risk genes of BD and was associated with BD in a GWAS comprising 682 patients with BD and 1,300 controls, as well as in a first follow-up step with 1,729 bipolar patients and 2,313 controls and a second follow-up step with a total of 6,030 patients and 31,749 controls $\left(p=2.14 \times 10^{-9}\right.$ in the combined analysis of all study samples) ${ }^{[48]}$.

ANK3 codes for Ankyrin 3, an adaptor protein, which regulates the assembly of voltage-gated sodium channels at axon initial segments and is therefore obviously of functional importance for psychiatric diseases. Already Ferreira and colleagues found in their 4,387 bipolar cases and 6,209 controls comprising whole genome study a strong association between the rs10994336 ANK3 SNP and $\operatorname{BD}\left(p=9.1 \times 10^{-9}\right)^{[42]}$. Consequently, the genome wide significant association of $A N K 3$ with BD was replicated in numerous GWAS ${ }^{[6,7,42-47]}$.

CACNA1C, the other mentioned well replicated BD susceptibility gene, codes for the alpha1C subunit of the L-type voltage-gated calcium channel. CACNA1C showed already a strong association with $\mathrm{BD}$ in the whole genome scan by Sklar and colleagues in $2008^{[53]}$, which included 1,461 bipolar participants and 2,008 controls. Also, a GWAS by Ferreira et al. showed a significant association of the rs1006737 CACNA1C (combined $p=7.0 \times 10^{-8}$ ) with BD in an overall sample of 4,387 bipolar cases and 6,209 controls ${ }^{[42]}$. This assumes that ion channel subunits and associated proteins play an important role in the pathogenesis of $\mathrm{BD}^{[42,54]}$. As mentioned, CACNA1C is also involved in schizophrenia and MDD ${ }^{[55-58]}$ and influences verbal fluency and memory ${ }^{[59,60]}$.

In the era of personalized medicine, there might even be the possibility that every single person with $\mathrm{BD}$ has a distinct kaleidoscope of ordinary SNPs, which is leading to the vulnerability for developing manic or depressed episodes. Nevertheless, the genetic analysis of special, narrowed endophenotypes as well as cross-disorder studies are necessary to elucidate the complex disease mechanisms of the $\mathrm{BD}$ spectrum and to further increase the knowledge about the disease. The next lines will include some of those narrowed subtypes.

\subsection{Lithium Response}

Lithium is still the golden standard of mood stabilization according to international guidelines. It prevents both 
affective episodes (mania and depression) and has even anti-suicidal effects ${ }^{[4]}$. Lithium interacts with diverse characteristic pathways of BD e.g., with the molecular $24 \mathrm{~h}$ clock by inhibition of GSK3Beta, which phosphorylates the gene products of the cycle length determining clock gene PER ${ }^{[61]}$. The latter is important, because the molecular clock is concatenated with mood, which can be observed clinically and molecular biologically. Disruptions of circadian rhythms like jetlag, night shifts or sleeping disorders lead to mood changes, which can be observed clinically and even in the general population. Those mood swings can be also explained with disturbed circadian rhythms on a molecular level. The complex clockwork of clock genes acts as transcriptional translational feedback loop and implements the important $24 \mathrm{~h}$ rhythm of diverse body functions (e.g., hormone secretion, regulation of body temperature or regulation of the heart beat). One further important function of the molecular clock is the transcription activation of the MAOA gene, which encodes for the enzyme Monoamine oxidase $A$ that is responsible for the breakdown of the neurotransmitter serotonin, noradrenaline and dopamine. The gene product of the clock gene ARNTL binds as heterodimer to E-boxes of MAOA and activates the transcription of the enzyme MAOA, which explains the strong interconnection between mood and the molecular clock $^{[37,38]}$.

Further research on Lithium-Genetics is tremendously important, because only around $30 \%$ of treated bipolar patients respond completely to lithium. The renown ConLiGen consortium (Consortium on Lithium Genetics) has been elucidating the genetic architecture of the lithium response pathways with PGRS analyses recently (Schizophrenia PGRS and Depression PGRS). First of all, Schizophrenia PGRS were analysed in a recent large-scale, collaborative ConLiGen GWAS-dataset (2,586 individuals with BD with a history of long-term lithium treatment). Weighted schizophrenia polygenic scores were calculated before in the international multicenter discovery GWAS-sample of 36,989 individuals with Schizophrenia. Schizophrenia PGRS (Polygenic Risk Score) as inversely associated with lithium response $\left(p<5 \times 10^{-2}\right)$, which is explaining $0.8 \%$ of the variance. Thus, patients with a low polygenic load for schizophrenia had a better response to lithium ${ }^{[5]}$. Furthermore, the lithium-response phenotype was also associated with inflammation and immunology pathways in the cross-trait meta-GWAS (15 genetic variants belonged to the HLA (human leukocyte antigens) region, the antigen presentation pathway and inflammatory cytokines e.g., TNF (Tumor Necrosis Factor), IL-4 (interleukin 4), IFNy (Interferon Y) $)^{[5]}$.

Second, the Depression PGRS was used in another recent analysis of the ConLiGen consortium to further distinguish between lithium responders and non-responders. Recent findings from the mentioned ConLiGen GWAS-data set ( $n=2,586$ bipolar patients) showed that the lithium response was better in bipolar patients (type I) with a low polygenic load for depressive traits compared to bipolar individuals with high polygenic load (MDD (Major Depressive Disorder): OR = 1.64 [95\% Cl: 1.26-2.15]). Summarized, the genetic load of depression traits in individuals with BD lower their probabilities to respond optimally to the mood stabilizer lithium ${ }^{[62]}$.

\subsection{Chronotypes}

The association between circadian rhythms and mood was also found in a recent GWAS of circadian rhythmicity in 71,500 UK Biobank participants and polygenic association with mood instability. Ferguson et al. made a large scale GWAS of the RA (relative amplitude), which is a parameter for restactivity cycles measured with an accelerometer in 71,500 study participants from the UK Biobank ${ }^{[39]}$. PGRS for low RA were used to investigate potential associations with psychiatric phenotypes (e.g., mood instability, MDD, neuroticism). The PGRS for low relative amplitude was significantly associated with mood instability (at PGRS threshold 0.05: OR $=1.02,95 \% \mathrm{Cl}=1.01-1.02, p=9.6 \times 10^{-5}$ ) and with MDD (at PGRS threshold 0.1: OR = 1.03, $95 \% \mathrm{Cl}=1.01-1.05, p=0.025)$ and neuroticism (at PGRS threshold 0.5: Beta $=0.02,95 \%$ $\mathrm{Cl}=0.007-0.04, p=0.021)$. Furthermore, two independent genetic loci were associated with Iow RA, within genomic regions for Neurofascin (NFASC) and Solute Carrier Family 25 Member 17 (SLC25A17). NFASC encodes for a cell adhesion protein, which interacts with diverse other proteins that anchor $\mathrm{Na}^{+}$channels to the intracellular backbone of neurons ${ }^{[39]}$. NFASC is also involved in brain developmental steps in the organization of axon initial segments, which also involves Ankyrin 3 (encoded by the BD risk gene $A N K 3^{[6,7,42-47]}$. The findings from Ferguson show that circadian rhythms are linked with genes that are important for generating action potentials, which are tremendously important for cognitive functioning. SLC25A17 encodes for a membrane protein, which belongs to the mitochondrial solute carriers. The SLC25A17 gene has been associated with Schizophrenia and adrenomyeloneuropathy, which shows diverse CNS (central nervous system) symptoms based on the accumulation of short chain fatty acids in the brain ${ }^{[39]}$.

\subsection{BD type I versus BD type II}

On a clinical level BD can be divided in BD type I and BD type II. The recent large-scale GWAS from the BD working group of the PGC showed also a distinct genetic pattern. BD type I has been strongly 
genetically correlated with schizophrenia while BD type II has been more strongly correlated with MDD, which shows that BD spectrum disorders provide a continuum and that the boundaries between psychiatric disorders are blurred and not as strict as Kraepelin had suggested ${ }^{[41]}$.

\subsection{Cross-Disorder Design}

Diverse genetic studies, either early linkage-, gene association studies, CNV (Copy Number Variation) analyses, as well as modern GWAS pointed toward a variety of shared SNPs and CNVs, as well as larger rare structural variants between diverse psychiatric diseases. There is an extensive list of shared genes between BD, MDD and schizophrenia that were analysed in the last decade by diverse approaches. Especially candidate genes involved in cognition pathways (e.g., BDNF, CACNA1C, COMT) are shared by disease mechanisms of diverse psychiatric diseases ${ }^{[6]}$. The PGC cross disorder group discovered in a sample comprising of five psychiatric disorders $(33,332$ cases and 27,888 controls) a genome wide association of CACNA1C and CACNB2 with psychiatric disorders, which underlines the importance of calcium signalling in psychiatric diseases ${ }^{[3,64]}$. Ruderfer et al. also found in a combined GWAS of 19,779 individuals with BD and schizophrenia versus 19,423 controls five already previously known genome-wide significant gene variants of CACNA1C, IFI44L, MHC, TRANK1 and MAD1L1, as well as one novel locus near PIK3C2A ${ }^{[65]}$. GWAS performed in CrossDisorder designs can therefore help to elucidate still hidden heritability. Nevertheless, also other forms of genetic variation should be considered. The history of the 22q11 microdeletion, which increases the risk for schizophrenia by the approximately 30 to 50 -fold ${ }^{[22]}$, underlines the importance of combining diverse molecular biological, genetic and epigenetic approaches to decipher the underlying pathomechanisms of psychiatric diseases on multifactorial levels (CNV analyses, classic Human Genetics approaches, epigenetics methods etc.). Large modern Cross-Disorder analyses of CNVs could replicate again diverse variants associated with BD and schizophrenia (1q21.1, 15q13.3, 16p11.2 and 22q11.21) ${ }^{[66]}$. To further solve the mystery about the biological underpinning of $\mathrm{BD}$ and schizophrenia diverse approaches are necessary, as well as narrowing and broadening (cross-disorder design) of the phenotype. Currently, the PGRS analysis is a method that is very promising for analyzing treatment response or for improving risk prediction ${ }^{[7]}$.

\subsection{Polygenic Risk Score for Schizophrenia in BD}

Furthermore, PGRS are helpful to analyse genetic overlaps of different psychiatric traits. The latter is rather important, because decades of genetic research elucidated that diverse psychiatric diseases share risk genes. BD shares risk genes with MDD, as well as schizophrenia ${ }^{[6,67]}$. Example given gene variants associated with synaptogenesis, growth hormones, neurotransmitter metabolism, signal transduction, ion channels or in general with "cognition pathways", as well as circadian rhythm genes were found to be shared between diverse psychiatric entities ${ }^{[7]}$. Nowadays, we know from Molecular Psychiatry that the boundaries between affective disorders and schizophrenia are more blurred than Kraepelin had expected; some authors even speak of the "psychosis continuum model" ${ }^{[65,68]}$. Summarized, there seems to be on one hand a variety of gene variants that are associated globally with psychiatric diseases e.g., involved in general cognition pathways and on the other hand a set of genes, which predisposes for a distinct phenotype. The PGRS provides an elegant method to predict traits in narrowed phenotypes and in Cross-Disorder-Samples ${ }^{[50]}$.

The polygenic risk score for schizophrenia can be used to detect traits with psychotic features ${ }^{[50,69]}$. Example given, the PGRS for schizophrenia can detect psychotic phenotypes in BD samples and has already been widely used by different working groups to distinguish between BD subtypes. Hamshere et al. found for example significantly higher Schizophrenia PGRS in BD with psychotic features in their study comprising the BD sample from the Wellcome Trust Case-Control Consortium (WTCCC) and the independent replication data is from the University College London (UCL). They used the PGRS to test whether schizophrenia polygenic risk alleles discriminate between bipolar patients with and without psychotic/schizoaffective features ${ }^{[70]}$. Matej Markota and colleagues analyzed if the genetic overlap between schizophrenia and BD was higher for $\mathrm{BD}$ with a history of manic psychosis compared to other BD subtypes. The polygenic risk score for schizophrenia was calculated in the Mayo Clinic Bipolar Biobank cohort (957 bipolar individuals with different histories of psychosis and 778 healthy controls) and was significantly higher for individuals with BD type I with manic psychosis ${ }^{[71]}$. Calafato and colleagues used, as mentioned before, the PGRS for schizophrenia to identify psychotic disorders ${ }^{[69]}$. The PGRS can also be used to investigate traits like age at onset e.g., Kalman and colleagues investigated the PGRS for schizophrenia and BD in synopsis with age at onset, but with negative results ${ }^{[72]}$. Groundbreaking results were also achieved by the PGC to either distinguish $\mathrm{BD}$ and schizophrenia and on the other hand to search for shared pathways- 114 genome-wide significant loci were shared between schizophrenia and $B D$ in a sample comprising of 53,555 individuals with BD or schizophrenia and 54,065 controls. The 
results suggested especially the involvement of synaptic and neuronal pathways in both diseases. Nevertheless, they found also genetic differences between schizophrenia and BD, which also underlines the genetic differences between both diseases ${ }^{[63]}$. Taken together, there is accumulating evidence from molecular biological and genetic studies that Kraepelin's dichotomy between manic depressive disease and schizophrenia is no longer existing in a strict manner, but that boundaries are blurred. Furthermore, polygenic risk scores for schizophrenia and bipolar disorder are also predictors of creativity ${ }^{[73]}$. The association between polygenic loading for BD and schizophrenia with creativity is certainly a positive resource and may also have therapeutic implications e.g., in cognitive behavioral therapy (cognitive restructuring and psychoeducation) ${ }^{[73]}$.

Clinical implications include personalized medicine, stratified treatment and prevention. The medical field of Psychiatry is still struggling to implement diagnostic blood markers like those implemented in other medical disciplines. Analyzing blood works belongs to routine procedures in the field of internal medicine and blood parameters (e.g., the inflammation parameter CRP (c-reactive protein)) are commonly used before changing treatment strategies. Even personalized, genetic testing is already established in diverse medical fields. Especially in the field of oncology genetic analyses are used to determine whether patients respond to a certain chemotherapeutic treatment option or not. Even though testing for CYP genotypes (e.g., CYP2C9, CYP2C19, CYP2D6) would be commercially available, they are not yet widely distributed in clinical practice at Psychiatry departments even though sophisticated, personalized treatment based on genetic analyses would save the patient's precious time and would reduce individual suffering based on better treatment response ${ }^{[74]}$. Hopefully, the prospering field of psychiatric genetics will help to improve treatment strategies in the field of pharmacogenetics and also in the field of prevention and genetic risk prediction.

\section{REFERENCES}

1. Rothenhäusler HB, Täschner KT. Kompendium Praktische Psychiatrie. Berlin: Springer. 2007. p. 1-558.

2. Mclntyre RS, Danilewitz M, Liauw SS, Kemp DE, Nguyen HT, Kahn LS, et al. Bipolar disorder and metabolic syndrome: an international perspective. J Affect Disord. 2010; 126(3): 366-387.

3. Mclntyre RS, Woldeyohannes HO, Soczynska JK, Miranda A, Lachowski A, Liauw SS, et al. The rate of metabolic syndrome in euthymic
There may also be a broad application field in regard of $P G R S$ analyses. Interestingly, PGRS for $\mathrm{BD}$ and schizophrenia could potentially also be used for prevention in the field of addiction. Reginsson et al. used the PGRS for schizophrenia and BD to predict addiction to nicotine, alcohol or drugs in study participant without psychotic disorders, which could help to prevent addiction (e.g., nicotine addiction) in patients who are at special risk ${ }^{[75]}$.

Most important is the fact that the PGRS can be used for treatment stratification to improve treatment response. The gained knowledge from the ConLiGen GWAS may help to develop stratified pharmacotherapeutic approaches in regard of lithium therapy. This will help to diminish the number of nonresponders based on personalized treatment, which would decrease on one hand individual suffering and would also reduce hospitalization rates ${ }^{[5]}$. PGRS may also help to detect cardiovascular risk in patients with BD or psychiatric diseases in general, which would improve prevention and would help to reduce cardiovascular mortality in individuals with psychiatric disorders. Prevention strategies against cardiovascular mortality is crucial for patients with $\mathrm{BD}$, because they show commonly comorbid obesity and metabolic syndrome ${ }^{[2,3]}$. Summarized, the Polygenic Risk Score is an important instrument for treatment decisions and a further step towards precision medicine.

\section{CONFLICTS OF INTEREST}

The authors declare that there is no conflict of interest regarding the publication of this paper.

\section{AUTHORS CONTRIBUTIONS}

Both authors contributed by writing this review. S.B. and E.R. planned the review, S.B. wrote the first draft. E.R. corrected and improved the first draft. Both improved the review according to the reviewer's suggestions.

Canadian individuals with bipolar I/II disorder. Adv Ther. 2010; 27(11): 828-836.

4. DGBS e.V. und DGPPN e.V. S3-Leitlinie zur Diagnostik und Therapie Bipolarer Störungen. Available online: http://www.leitlinie-bipolar.de/wpcontent/uploads/2016/07/S3_Leitlinie-Bipolar_ V1_8.pdf (accessed on 18 December 2018).

5. International Consortium on Lithium Genetics (ConLi+Gen), Amare AT, Schubert KO, Hou L, Clark SR, Papiol S, et al. Association 
of Polygenic Score for Schizophrenia and HLA Antigen and Inflammation Genes With Response to Lithium in Bipolar Affective Disorder: A Genome-Wide Association Study. JAMA Psychiatry. 2018; 75(1): 65-74.

6. Bengesser S, Reininghaus E. Genetics of Bipolar Disorder. Bern: Peter-Lang; 2013.

7. Schulze TG, McMahon FJ, editors. Psychiatric Genetics: A Primer for Clinical and Basic Scientists. 1st ed. New York: Oxford University Press; 2018.

8. Egeland JA, Gerhard DS, Pauls DL, Sussex $\mathrm{JN}$, Kidd KK, Allen CR, et al. Bipolar affective disorders linked to DNA markers on chromosome 11. Nature. 1987; 325(6107): 783-787.

9. Kirschenbaum B, Goldman SA. Brain-derived neurotrophic factor promotes the survival of neurons arising from the adult rat forebrain subependymal zone. Proc Natl Acad Sci U S A. 1995; 92(1): 210-214.

10. Martinowich K, Lu B. Interaction between BDNF and serotonin: role in mood disorders. Neuropsychopharmacology. 2008; 33(1): 73-83.

11. Pencea V, Bingaman KD, Wiegand SJ, Luskin $M B$. Infusion of brain-derived neurotrophic factor into the lateral ventricle of the adult rat leads to new neurons in the parenchyma of the striatum, septum, thalamus, and hypothalamus. J Neurosci. 2001; 21(17): 6706-6717.

12. Schumacher J, Jamra RA, Becker T, Ohlraun $\mathrm{S}$, Klopp N, Binder EB, et al. Evidence for a relationship between genetic variants at the brain-derived neurotrophic factor (BDNF) locus and major depression. Biol Psychiatry. 2005; 58(4): 307-314.

13. Sklar $P$, Gabriel SB, Mclnnis MG, Bennett $P$, Lim YM, Tsan G, et al. Family-based association study of 76 candidate genes in bipolar disorder: BDNF is a potential risk locus. Brain-derived neutrophic factor. Mol Psychiatry. 2002; 7(6): 579-593.

14. Zigova T, Pencea V, Wiegand SJ, Luskin MB. Intraventricular administration of BDNF increases the number of newly generated neurons in the adult olfactory bulb. Mol Cell Neurosci. 1998; 11(4): 234-245.

15. Binder DK, Scharfman HE. Brain-derived neurotrophic factor. Growth Factors 2004; 22(3): 123-131.

16. Mamounas LA, Blue ME, Siuciak JA, Altar CA. Brain-derived neurotrophic factor promotes the survival and sprouting of serotonergic axons in rat brain. J Neurosci. 1995; 15(12): 7929-7939.
17. Rybakowski JK. BDNF gene: functional Val66Met polymorphism in mood disorders and schizophrenia. Pharmacogenomics. 2008; 9(11): 1589-1593.

18. Le-Niculescu H, Patel SD, Bhat M, Kuczenski $\mathrm{R}$, Faraone SV, Tsuang MT, et al. Convergent functional genomics of genome-wide association data for bipolar disorder: comprehensive identification of candidate genes, pathways and mechanisms. Am J Med Genet B Neuropsychiatr Genet. 2009; 150B(2): 155-181.

19 Lasky-Su JA, Faraone SV, Glatt SJ, Tsuang MT. Meta-analysis of the association between two polymorphisms in the serotonin transporter gene and affective disorders. Am J Med Genet B Neuropsychiatr Genet. 2005; 133B(1): 110-115.

20. Heils A, Teufel A, Petri S, Stober G, Riederer P, Bengel $D$, et al. Allelic variation of human serotonin transporter gene expression. J Neurochem. 1996; 66(6): 2621-2624.

21. Hoefgen B, Schulze TG, Ohlraun $S$, von Widdern O, Hofels S, Gross M, et al. The power of sample size and homogenous sampling: association between the 5-HTTLPR serotonin transporter polymorphism and major depressive disorder. Biol Psychiatry. 2005; 57(3): 247-251.

22. Murphy KC, Jones LA, Owen MJ. High rates of schizophrenia in adults with velo-cardio-facial syndrome. Arch Gen Psychiatry. 1999; 56(10): 940-945.

23. Black GC, Chen ZY, Craig IW, Powell JF. Dinucleotide repeat polymorphism at the MAOA locus. Nucleic Acids Res. 1991; 19(3): 689.

24. Furlong RA, Ho L, Rubinsztein JS, Walsh C, Paykel ES, Rubinsztein DC. Analysis of the monoamine oxidase A (MAOA) gene in bipolar affective disorder by association studies, metaanalyses, and sequencing of the promoter. Am J Med Genet. 1999; 88(4): 398-406.

25. Kawada Y, Hattori M, Dai XY, Nanko S. Possible association between monoamine oxidase $A$ gene and bipolar affective disorder. Am J Hum Genet 1995; 56(1): 335-336.

26. Rubinsztein DC, Leggo J, Goodburn S, Walsh C, Jain S, Paykel ES. Genetic association between monoamine oxidase A microsatellite and RFLP alleles and bipolar affective disorder: analysis and meta-analysis. Hum Mol Genet. 1996; 5(6): 779-782.

27. Lim LC, Powell J, Sham P, Castle D, Hunt N, Murray $\mathrm{R}$, et al. Evidence for a genetic association between alleles of monoamine oxidase A gene and bipolar affective disorder. Am J Med Genet. 1995; 60(4): 325-331. 
28. Preisig M, Bellivier F, Fenton BT, Baud P, Berney $A$, Courtet $P$, et al. Association between bipolar disorder and monoamine oxidase A gene polymorphisms: results of a multicenter study. Am J Psychiatry. 2000; 157(6): 948-955.

29. Muller DJ, Serretti A, Sicard T, Tharmalingam S, King N, Artioli P, et al. Further evidence of MAO-A gene variants associated with bipolar disorder. Am J Med Genet B. 2007; 144B(1): 37-40.

30. Le-Niculescu H, McFarland MJ, Ogden CA, Balaraman Y, Patel S, Tan J, et al. Phenomic, convergent functional genomic, and biomarker studies in a stress-reactive genetic animal model of bipolar disorder and co-morbid alcoholism. Am J Med Genet B. 2008; 147B(2): 134-166.

31. Nievergelt CM, Kripke DF, Barrett TB, Burg E, Remick RA, Sadovnick AD, et al. Suggestive evidence for association of the circadian genes PERIOD3 and ARNTL with bipolar disorder. Am J Med Genet B 2006; 141B(3): 234-241.

32. Kripke DF, Nievergelt CM, Joo E, Shekhtman T, Kelsoe JR. Circadian polymorphisms associated with affective disorders. J Circadian Rhythms. 2009; 7: 2 .

33. Mansour HA, Talkowski ME, Wood J, Chowdari KV, McClain L, Prasad K, et al. Association study of 21 circadian genes with bipolar I disorder, schizoaffective disorder, and schizophrenia. Bipolar Disord. 2009; 11(7): 701-710.

34. Mansour HA, Wood J, Logue T, Chowdari KV, Dayal M, Kupfer DJ, et al. Association study of eight circadian genes with bipolar I disorder, schizoaffective disorder and schizophrenia. Genes Brain Behav. 2006; 5(2): 150-157.

35. Shi J, Wittke-Thompson JK, Badner JA, Hattori E, Potash JB, Willour VL, et al. Clock genes may influence bipolar disorder susceptibility and dysfunctional circadian rhythm. Am J Med Genet B Neuropsychiatr Genet. 2008; 147B(7): 10471055.

36. Hosak L. Role of the COMT gene Val158Met polymorphism in mental disorders: a review. Eur Psychiatry. 2007; 22(5): 276-281.

37. Hampp G, Ripperger JA, Houben T, Schmutz I, Blex C, Perreau-Lenz S, et al. Regulation of monoamine oxidase $A$ by circadian-clock components implies clock influence on mood. Curr Biol. 2008; 18(9): 678-683.

38. Bengesser SA, Reininghaus EZ, Lackner N, Birner A, Fellendorf FT, Platzer M, et al. Is the molecular clock ticking differently in bipolar disorder? Methylation analysis of the clock gene ARNTL. World J Biol Psychiatry. 2016; 14: 1-9.
39. Ferguson A, Lyall LM, Ward J, Strawbridge RJ, Cullen B, Graham N, et al. Genome-Wide Association Study of Circadian Rhythmicity in 71,500 UK Biobank Participants and Polygenic Association with Mood Instability. EbioMedicine. 2018; 35: 279-287.

40. Sklar P, Ripke S, Scott LJ, Andreassen OA, Cichon S, Craddock N, et al. Large-scale genome-wide association analysis of bipolar disorder identifies a new susceptibility locus near ODZ4. Nat Genet. 2011; 43(10): 977-983.

41. Eli Stahl, Gerome Breen, Andreas Forstner, Andrew McQuillin, Stephan Ripke, Bipolar Disorder Working Group of the Psychiatric Genomics Consortium, et al. Genomewide association study identifies 30 loci associated with bipolar disorder. Available online: https:// www.biorxiv.org/content/early/2018/01/24/173062 (accessed on 7th of December 2018).

42. Ferreira MA, O'Donovan MC, Meng YA, Jones IR, Ruderfer DM, Jones L, et al. Collaborative genome-wide association analysis supports a role for ANK3 and CACNA1C in bipolar disorder. Nat Genet. 2008; 40(9): 1056-1058.

43. Baum AE, Hamshere M, Green E, Cichon S, Rietschel M, Noethen MM, et al. Meta-analysis of two genome-wide association studies of bipolar disorder reveals important points of agreement. Mol Psychiatry. 2008; 13(5): 466-467.

44. Segurado R, Detera-Wadleigh SD, Levinson DF, Lewis CM, Gill M, Nurnberger JI, Jr, et al. Genome scan meta-analysis of schizophrenia and bipolar disorder, part III: Bipolar disorder. Am J Hum Genet. 2003; 73(1): 49-62.

45. Scott LJ, Muglia P, Kong XQ, Guan W, Flickinger $\mathrm{M}$, Upmanyu $\mathrm{R}$, et al. Genome-wide association and meta-analysis of bipolar disorder in individuals of European ancestry. Proc Natl Acad Sci U S A. 2009; 106(18): 7501-7506.

46. Schulze TG, Detera-Wadleigh SD, Akula N, Gupta A, Kassem L, Steele J, et al. Two variants in Ankyrin 3 (ANK3) are independent genetic risk factors for bipolar disorder. Mol Psychiatry. 2009; 14(5): 487-491.

47. Takata A, Kim SH, Ozaki N, Iwata N, Kunugi H, Inada $\mathrm{T}$, et al. Association of ANK3 with bipolar disorder confirmed in East Asia. Am J Med Genet B. 2011; 156B(3): 312-315.

48. Cichon S, Muhleisen TW, Degenhardt FA, Mattheisen M, Miro X, Strohmaier J, et al. Genome-wide association study identifies genetic variation in neurocan as a susceptibility factor for bipolar disorder. Am J Hum Genet. 2011; 88(3): 372-381. 
49. Dudbridge F. Power and predictive accuracy of polygenic risk scores. PLoS Genet. 2013; 9(3): e1003348.

50. Maier R, Moser G, Chen GB, Ripke S. CrossDisorder Working Group of the Psychiatric Genomics Consortium, Coryell W, et al. Joint analysis of psychiatric disorders increases accuracy of risk prediction for schizophrenia, bipolar disorder, and major depressive disorder. Am J Hum Genet. 2015; 96(2): 283-294.

51. Purcell SM, Moran JL, Fromer M, Ruderfer D, Solovieff N, Roussos $\mathrm{P}$, et al. A polygenic burden of rare disruptive mutations in schizophrenia. Nature. 2014; 506(7487): 185-190.

52. Schultz CC, Muhleisen TW, Nenadic I, Koch K, Wagner G, Schachtzabel C, et al. Common variation in NCAN, a risk factor for bipolar disorder and schizophrenia, influences local cortical folding in schizophrenia. Psychol Med. 2014; 44(4): 811-820.

53. Sklar P, Smoller JW, Fan J, Ferreira MA, Perlis $\mathrm{RH}$, Chambert $\mathrm{K}$, et al. Whole-genome association study of bipolar disorder. Mol Psychiatry. 2008; 13(6): 558-569.

54. Askland K, Read C, Moore J. Pathways-based analyses of whole-genome association study data in bipolar disorder reveal genes mediating ion channel activity and synaptic neurotransmission. Hum Genet. 2009; 125(1):63-79.

55. Casamassima F, Huang J, Fava M, Sachs GS, Smoller JW, Cassano GB, et al. Phenotypic effects of a bipolar liability gene among individuals with major depressive disorder. Am J Med Genet B. 2010; 153B(1): 303-309.

56. Green EK, Grozeva D, Jones I, Jones L, Kirov G, Caesar S, et al. The bipolar disorder risk allele at CACNA1C also confers risk of recurrent major depression and of schizophrenia. Mol Psychiatry. 2010; 15(10): 1016-1022.

57. Moskvina V, Craddock N, Holmans P, Nikolov I, Pahwa JS, Green E, et al. Gene-wide analyses of genome-wide association data sets: evidence for multiple common risk alleles for schizophrenia and bipolar disorder and for overlap in genetic risk. Mol Psychiatry. 2009; 14(3): 252-260.

58. Schizophrenia Psychiatric Genome-Wide Association Study (GWAS) Consortium. Genome-wide association study identifies five new schizophrenia loci. Nat Genet. 2011; 43(10): 969-976.

59. Erk S, Meyer-Lindenberg A, Schnell K, Opitz von Boberfeld C, Esslinger C, Kirsch P, et al. Brain function in carriers of a genome-wide supported bipolar disorder variant. Arch Gen Psychiatry. 2010; 67(8): 803-811.

60. Krug A, Nieratschker V, Markov V, Krach S, Jansen A, Zerres $\mathrm{K}$, et al. Effect of CACNA1C rs1006737 on neural correlates of verbal fluency in healthy individuals. Neuroimage. 2010; 49(2): 1831-1836.

61. Bhat RV, Budd Haeberlein SL, Avila J. Glycogen synthase kinase 3: a drug target for CNS therapies. J Neurochem. 2004; 89(6): 1313-1317.

62. Azmeraw T. Amare, Klaus Oliver T, Schubert International Consortium on Lithium Genetics (ConLi+Gen), Thomas G. Schulze, Bernhard T Baune. Polygenic scores for major depressive disorder and depressive symptoms predict response to lithium in patients with bipolar disorder. Available from: https://www.biorxiv.org/ content/early/2018/10/26/449363 (accessed on 7th of December 2018).

63. Bipolar Disorder and Schizophrenia Working Group of the Psychiatric Genomics Consortium, Bipolar Disorder and Schizophrenia Working Group of the Psychiatric Genomics Consortium. Genomic Dissection of Bipolar Disorder and Schizophrenia, Including 28 Subphenotypes. Cell. 2018; 173(7): 1705-1715.

64. Cross-Disorder Group of the Psychiatric Genomics Consortium. Identification of risk loci with shared effects on five major psychiatric disorders: a genome-wide analysis. Lancet. 2013; 381(9875): 1371-1379.

65. Ruderfer DM, Fanous AH, Ripke S, McQuillin A, Amdur RL, Schizophrenia Working Group of the Psychiatric Genomics Consortium, et al. Polygenic dissection of diagnosis and clinical dimensions of bipolar disorder and schizophrenia. Mol Psychiatry. 2014; 19(9): 1017-1024.

66. Chen J, Calhoun VD, Perrone-Bizzozero NI, Pearlson GD, Sui J, Du Y, et al. A pilot study on commonality and specificity of copy number variants in schizophrenia and bipolar disorder. Transl Psychiatry. 2016; 6(5): e824.

67. International Schizophrenia Consortium, Purcell SM, Wray NR, Stone JL, Visscher PM, O'Donovan $\mathrm{MC}$, et al. Common polygenic variation contributes to risk of schizophrenia and bipolar disorder. Nature. 2009; 460(7256): 748-752.

68. Tesli M, Espeseth T, Bettella F, Mattingsdal M, Aas M, Melle I, et al. Polygenic risk score and the psychosis continuum model. Acta Psychiatr Scand. 2014; 130(4): 311-317.

69. Calafato MS, Thygesen JH, Ranlund S, Zartaloudi E, Cahn W, Crespo-Facorro B, et al. Use of 
schizophrenia and bipolar disorder polygenic risk scores to identify psychotic disorders. $\mathrm{Br} \mathrm{J}$ Psychiatry. 2018; 213(3): 535-541.

70. Hamshere ML, O'Donovan MC, Jones IR, Jones L, Kirov G, Green EK, et al. Polygenic dissection of the bipolar phenotype. Br J Psychiatry. 2011; 198(4): 284-288.

71. Markota M, Coombes BJ, Larrabee BR, McElroy $\mathrm{SL}$, Bond DJ, Veldic M, et al. Association of schizophrenia polygenic risk score with manic and depressive psychosis in bipolar disorder. Transl Psychiatry. 2018; 8(1): 188.

72. Kalman JL, Papiol S, Forstner AJ, Heilbronner U, Degenhardt F, Strohmaier J, et al. Investigating polygenic burden in age at disease onset in bipolar disorder: Findings from an international multicentric study. Bipolar Disord. 2018. doi: 10.1111/bdi.12659.

73. Power RA, Steinberg S, Bjornsdottir G, Rietveld CA, Abdellaoui A, Nivard MM, et al. Polygenic risk scores for schizophrenia and bipolar disorder predict creativity. Nat Neurosci. 2015; 18(7): 953-955.

74. Bousman C, Maruf AA, Muller DJ. Towards the integration of pharmacogenetics in psychiatry: a minimum, evidence-based genetic testing panel. Curr Opin Psychiatry. 2019; 32(1): 7-15.

75. Reginsson GW, Ingason A, Euesden J, Bjornsdottir G, Olafsson S, Sigurdsson E, et al. Polygenic risk scores for schizophrenia and bipolar disorder associate with addiction. Addict Biol. 2018; 23(1): 485-492. 